\title{
Acknowledgement to MIR Board Members and Ad hoc Reviewers
}

(c) Springer-Verlag GmbH Germany, part of Springer Nature 2020

We would like to thank all members of MIR's Editorial Board and Editorial Review Board for their valuable inputs they have provided in the year 2019 to further advance the journal. Furthermore, we would like to thank the following scholars who supported MIR in the last year as ad-hoc reviewers:

Elizabeth Alexander, Farzad Alvi, Ulf Andersson, Preet S. Aulakh, Anne Bachmann, Yongjian Bao, Matthias Baum, Florian Becker-Ritterspach, Christina Bettinelli, Arjun Bhardwaj, Torsten Biemann, Torsten Bornemann, Suleika Bort, Narjess Boubakri, Keith Brouthers, Bruhns, Nealia S. Bruning, Anne Burmeister, Steve Burt, Frank Calisse, Francesco Ciabuschi, Joseph A. Clougherty, Luis Alfonso Dau, Tobias Dauth, John Dawson, Desislava Dikova, Pavlos Dimitratos, Christoph Dörrenbächer, Rian Drogendijk, Mengqiao Du, Gunter Dufey, Stefan Eckert, Peter Enderwick, Prescott C. Ensign, Ricardo Flores, Cher-Min Fong, Johann Fortwengel, Jörg Freiling, Fabian Froese, Manfred Fuchs, Oliver Furrer, Mika Gabrielsson, Hanna Gajewska-De Mattos, Jens Gammelgaard, Keith Glaister, Siegfried Gudergan, Igor Gurkov, Amjad Hadjikhani, Arno Haslberger, Melanie Hassett, Louis Hébert, Martin Hemmert, Martin Hoegl, Ulf Holm, Linda Hsieh, Kuo-Feng Huang, Kate Hutchings, Rodrigo Isidor, Marshall S. Jiang, Jae Jung, Mario Kafouros, Philip Kappen, Alexei Koveshnikov, Jochem Kroezen, Christina Kühl, Olli Kuivalainen, Thomas Lawton, Tanja Leppäaho, Jiatao Li, Xiaoying Li, Leonardo Liberman-Yaconi, Ru-Shiun Liou, Xiaming Liu, Christian Lüthje, Antonio Majocchi, Shige Makino, Mary M. Maloney, Valentina Marano, Gaia Melloni, Klaus Meyer, Michael Moffett, Fiona Moore, Michael MüllerCamen, Etayankara Muralidharan, William Newburry, Quyen Nguyen, Niina Nummela, Claude Obadia, Robert Ostergard, Marina Papanastassiou, Ronaldo Parente, Byung Il Park, Pasquale Massimo Picone, José Pla Barber, Skylar Powell, Shameen Prashantham, Vivien Procher, Jonas Puck, Larissa Rabbiosi, Kannan Ramaswamy, Olaf N. Rank, Sougata Ray, Sebastian Reiche, Bjoern Röber, Alicia Rodriguez, Thomas W. Roehl, Björn Schmeisser, Andreas Schotter, Christian Schwens, Rudolf R. Sinkovics, Carl Arthur Solberg, Colette Southam, Thomas Steger, Dennis Steininger, Roger Strange, Yama Temouri, Helene Tenzer, Georg Trautnitz, Anja Tuschke, Florian Überbacher, Hinrich Voss, Chengqi Wang, George O. White, Yingqi Wie, Robert Wilken, Christopher Williams, Kai Xu, Mo Yamin, Yong Yang, Attila Yaprak, Emre Yildiz, Wenlong Yuan, Florian Zapkau, Yuping Zeng, Miriam Zschoche.

Michael-Jörg Oesterle (Co-Editor in Chief) Joachim Wolf (Co-Editor in Chief)

Publisher's Note Springer Nature remains neutral with regard to jurisdictional claims in published maps and institutional affiliations. 